\title{
Coexistence of the electron Cooper pair and antiferromagnetic short-range correlation in copper oxide materials
}

\author{
Shiping Feng \\ CCAST (World Laboratory) P. O. Box 8730, Beijing 100080, China \\ Department of Physics, Beijing Normal University, Beijing 100875, China \\ Institute of Theoretical Physics, Academia Sinica, Beijing 100080, China
}

\begin{abstract}
Within the fermion-spin theory, the physical properties of the electron pairing state in the copper oxide materials are discussed. According to the common form of the electron Cooper pair, it is shown that there is a coexistence of the electron Cooper pair and magnetic short-range correlation, and hence the antiferromagnetic short-range correlation can persist into the superconducting state. Moreover, the mean-field results indicate that the electron pairing state originating from the pure magnetic interaction in the two-dimensional $t$ - $J$ model is the local state, and then does not reveal the true superconducting ground-state.
\end{abstract}

71.20.Cf, 74.72.Hs, 79.60.Bm

After over ten years of intense experimental studies of the copper oxide superconductors, a significant body of reliable and reproducible data has been accumulated by using many probes, which show that the properties of the copper oxide superconductors can be explained in terms of the electron pairing theory ${ }^{1,2}$. The experimental evidence includes the factor of $2 e$ occurring in the flux quantum and in the Josephson effect, as well as the electrodynamic and thermodynamic properties of the copper oxide superconductors ${ }^{3}$. The experimental results from the angle resolved photoemission spectroscopy imply that in the real space the gap function and pairing force have a range of one lattice spacing ${ }^{4,5}$. This is much different from the superconductivity in the conventional metals, where requires pairing with the longrange phase coherence ${ }^{6}$. Moreover, the unusual normal state properties of the copper oxide superconductors are also markedly different from those of the conventional superconductors and are usually viewed as manifestations of strong electron-electron correlations ${ }^{7,8}$, which cause the antiferromagnetic (AF) long-range-order (AFLRO) state in the undoped copper oxide materials. Although this AFLRO disappears for the hole doping concentration exceeds some critical value, the short-range AF correlation still persist even into the superconducting state in the underdoped and optimal doped regimes, which show that the short-range AF correlation may play a role in both the unconventional normal state properties and the mechanism of the superconductivity of the copper oxide materials ${ }^{7,8}$. Therefore there is the microscopic difference between the conventional superconductors and copper oxide superconductors, namely they have a different origin. It is believed that the correct theory for describing the anomalous properties of the copper oxide materials should involve the charge-spin separation in some form ${ }^{9}$.

Many researchers ${ }^{10}$ have argued successfully that the $t-J$ model provides a consistent description of the physical properties of the copper oxide materials. On the other hand, there is a lot of evidence from the experiments and numerical simulations in favour of the $t-J$ model as the basic underlying microscopic model ${ }^{7,8,11}$. In order to ac- count for the real experiments based on the $t-J$ model, it is crucial to impose the electron single occupancy onsite local constraint ${ }^{12}$. To satisfy this local constraint in analytical calculations, the fermion-spin theory has been proposed $^{13}$ to study the $t-J$ model. In this approach, the physical electron is decomposed into a spinless fermion and a hard-core boson, and then naturally incorporates the physics of the charge-spin separation. Within this approach, it has been shown ${ }^{14}$ that AFLRO in copper oxide materials vanishes around doping $\delta=5 \%$. The mean-field theory in the underdoped and optimal doped regimes without AFLRO has been developed ${ }^{15}$ to study the photoemission spectroscopy and electron dispersion. Moreover, the charge dynamics ${ }^{16}$ and spin dynamics ${ }^{17}$ in the normal state of the copper oxide materials have been discussed by considering fluctuations around this mean-field approximation (MFA), and the results are in qualitative agreement with the experiments and numerical simulations. In this paper, we apply this successful approach to discuss the physical property of the electron pairing state in the copper oxide materials. According to the common form of the electron Cooper pair, we show that there is a coexistence of the electron Cooper pair and AF short-range correlation, and hence the AF short-range magnetic fluctuation can persist into the superconducting state. Moreover, it is shown within the mean-field level that the singlet pair of electrons originating from the pure magnetic interaction in the twodimensional (2D) $t-J$ model is local, and then does not reveal the true superconducting ground-state.

According to the fermion-spin formulism ${ }^{13}$, the constrained electron operators in the $t-J$ model can be decomposed as, $C_{i \uparrow}=h_{i}^{\dagger} S_{i}^{-}, C_{i \downarrow}=h_{i}^{\dagger} S_{i}^{+}$with the spinless fermion operator $h_{i}$ keeping track of the charge (holon), while the pseudospin operator $S_{i}$ keeping track of the spin (spinon). In this representation, the $t-J$ model can be expressed ${ }^{13}$ as,

$$
H=t \sum_{\langle i j\rangle} h_{j}^{\dagger} h_{i}\left(S_{i}^{+} S_{j}^{-}+S_{i}^{-} S_{j}^{+}\right)-\mu \sum_{i} h_{i}^{\dagger} h_{i}
$$




$$
+J \sum_{\langle i j\rangle}\left(h_{i} h_{i}^{\dagger}\right)\left(\mathbf{S}_{i} \cdot \mathbf{S}_{j}-\frac{1}{4}\right)\left(h_{j} h_{j}^{\dagger}\right) .
$$

We treat this Hamiltonian within the MFA by introducing the following order parameters: $\chi=\left\langle S_{i}^{+} S_{i+\eta}^{-}\right\rangle$, $\chi_{z}=\left\langle S_{i}^{z} S_{i+\eta}^{z}\right\rangle, \phi_{\eta}=\left\langle h_{i}^{\dagger} h_{i+\eta}\right\rangle$, and $\Delta_{\eta}^{(h)}=\left\langle h_{i} h_{i+\eta}\right\rangle$ with $\eta= \pm \hat{x}, \pm \hat{y}$. $\chi$ and $\chi_{z}$ describe the spinon pair correlation, $\phi_{\eta}$ is the holon particle-hole parameter, while $\Delta_{\eta}^{(h)}$ represents the holon pair correlation. In this case, the $t-J$ Hamiltonian (1) can be decoupled as $H=H_{t}+$ $H_{J}-8 N t \chi \phi-4 N J_{e f f}\left(\chi+\chi_{z}\right)-4 N V_{e f f}\left(\left|\phi_{\eta}\right|^{2}-\left|\Delta_{\eta}\right|^{2}\right)$ with

$$
\begin{aligned}
H_{t} & =2 \sum_{i, \eta}\left(\chi t+V_{e f f} \phi_{\eta}\right) h_{i+\eta}^{\dagger} h_{i}-\mu \sum_{i} h_{i}^{\dagger} h_{i} \\
& -V_{\text {eff }} \sum_{i, \eta}\left(\Delta_{\eta}^{(h)} h_{i}^{\dagger} h_{i+\eta}^{\dagger}+\Delta_{\eta}^{(h) *} h_{i+\eta} h_{i}\right), \\
H_{J} & =\frac{1}{2} J_{e f f} \epsilon \sum_{i, \eta}\left(S_{i}^{+} S_{i+\eta}^{-}+S_{i}^{-} S_{i+\eta}^{+}\right) \\
& +J_{\text {eff }} \sum_{i, \eta} S_{i}^{z} S_{i+\eta}^{z},
\end{aligned}
$$

where $N$ is the number of sites, the doping dependent magnetic exchange energy $J_{\text {eff }}=J\left[(1-\delta)^{2}-\right.$ $\left.\phi_{\eta}^{2}+\left|\Delta_{\eta}^{(h)}\right|^{2}\right]$, the holon's effective attractive interaction $V_{e f f}=-J\left(\chi+\chi_{z}-1 / 4\right)$, and $\epsilon=1+2 t \phi_{\eta} / J_{e f f}$. Since the holon order parameters $\phi_{\eta}$ and $\Delta_{\eta}^{(h)}$ are decoupled from the term $V_{\text {eff }} \sum_{i, \eta} h_{i} h_{i}^{\dagger} h_{i+\eta} h_{i+\eta}^{\dagger}$ in the MFA, then there are many ways to choose the gauge for these order parameters $\phi_{\eta}$ and $\Delta_{\eta}^{(h)}$. However, in the copper oxide superconductors, some experiments seem consistent with an s-wave pairing ${ }^{2}$, while other measurements gave the evidence in favor of the $d$-wave pairing ${ }^{1}$. Therefore in the following discussions, we only consider the cases of the swave pairing $\phi_{y}=\phi_{x}=\phi, \Delta_{y}^{(h)}=\Delta_{x}^{(h)}=\Delta_{h}^{(s)}$, and the d-wave pairing $\phi_{y}=\phi_{x}=\phi, \Delta_{y}^{(h)}=-\Delta_{x}^{(h)}=\Delta_{h}^{(d)}$, respectively. In this case, the spinon mean-field Green's function $D\left(i-j, t-t^{\prime}\right)=\left\langle\left\langle S_{i}^{+}(t) ; S_{j}^{-}\left(t^{\prime}\right)\right\rangle\right\rangle$ of the Hamiltonian (3) have been discussed in detail in Ref. ${ }^{15}$ based on the Tyablikov ${ }^{18}$ and Kondo and Yamaji ${ }^{19}$ scheme. For the convenience of the further discussions in this paper, this spinon Green's function is rewritten here,

$$
\begin{aligned}
D(\mathbf{k}, \omega) & =\frac{\Lambda\left[\left(2 \epsilon \chi_{z}+\chi\right) \gamma_{k}-\left(\epsilon \chi+2 \chi_{z}\right)\right]}{2 \omega(k)} \\
& \times\left(\frac{1}{\omega-\omega(k)}-\frac{1}{\omega+\omega(k)}\right),
\end{aligned}
$$

where $\Lambda=8 J_{\text {eff }}, \gamma_{\mathbf{k}}=\left(\cos k_{x}+\cos k_{y}\right) / 2$, and the spinon spectrum $\omega(k)$ has been given in Ref. ${ }^{15}$. While the holon mean-field Hamiltonian (2) can be diagonalized by the Bogoliubov transformation in the momentum space, and then the holon mean-field Green's function $g\left(\mathbf{k}, t-t^{\prime}\right)=$ $\left\langle\left\langle h_{k}(t) ; h_{k}^{\dagger}\left(t^{\prime}\right)\right\rangle\right\rangle$ and holon mean-field anomalous Green's function $\Im_{h}\left(\mathbf{k}, t-t^{\prime}\right)=-\left\langle\left\langle h_{-k}(t) ; h_{k}\left(t^{\prime}\right)\right\rangle\right\rangle$ are obtained as

$$
\begin{aligned}
g(\mathbf{k}, \omega) & =\frac{1}{2}\left(1+\frac{\xi_{k}}{E_{k}}\right) \frac{1}{\omega-E_{k}} \\
& +\frac{1}{2}\left(1-\frac{\xi_{k}}{E_{k}}\right) \frac{1}{\omega+E_{k}} \\
\Im_{h}(\mathbf{k}, \omega) & =-\frac{\Delta_{h}^{(a)}(k)}{2 E_{k}}\left(\frac{1}{\omega-E_{k}}-\frac{1}{\omega+E_{k}}\right),
\end{aligned}
$$

respectively, where the holon spectrum $\xi_{k}=8(\chi t+$ $\left.\phi V_{e f f}\right) \gamma_{k}-\mu$, and the quasi-particle excitation energy $E_{k}=\sqrt{\xi_{k}^{2}+\left[\Delta_{h}^{(a)}(k)\right]^{2}}$. The gap function $\Delta_{h}^{(a)}(k)=$ $8 V_{\text {eff }} \Delta_{h}^{(a)} \gamma_{k}^{(a)}$ with $\Delta_{h}^{(a)}=\Delta_{h}^{(s)}, \gamma_{k}^{(a)}=\gamma_{k}^{(s)}=\gamma_{k}$ for s-wave pairing, and $\Delta_{h}^{(a)}=\Delta_{h}^{(d)}, \gamma_{k}^{(a)}=\gamma_{k}^{(d)}=$ $\left(\cos k_{x}-\cos k_{y}\right) / 2$ for $\mathrm{d}$-wave pairing, respectively, and satisfy the following equation,

$$
\Delta_{h}^{(a)}=\frac{1}{2 N} \sum_{k} \gamma_{k} \frac{\Delta_{h}^{(a)}(k)}{E_{k}} \operatorname{th}\left[\frac{1}{2} \beta E_{k}\right] .
$$

This gap equation must be solved simultaneously with other self-consistent equations ${ }^{15}$, therefore the holon pair transition temperature $T_{(h) c}^{(a)}$ is determined by the condition $\Delta_{h}^{(a)}\left(T_{(h) c}^{(a)}\right)=0$.

Before discussing the properties of the electron pairing state, we first consider the properties of the holon pairs. Although it is not observable from experiments, its feature will affect the properties of the physical electrons because of the strong interaction between holons and spinons within the framework of the charge-spin separation. Fig. 1 shows the results of the holon gap parameter in the s-wave symmetry $\Delta_{h}^{(s)}$ (solid line) and dwave symmetry $\Delta_{h}^{(d)}$ (dashed line) as a function of dopings at the temperature $T=0$ for (a) $t / J=2$ and (b) $t / J=2.5$, where holons favour the s-wave pairing at low dopings and d-wave pairing at high dopings. $\Delta_{h}^{(s)}\left(\Delta_{h}^{(d)}\right)$ is decreased (increased) with increasing dopings, and the value of these gap parameters is rather sensitive to the parameter $t / J$ and always decreased with increasing $t / J$. It is surprised that the ranges of the holon s-wave pairing state and holon d-wave pairing state are also strong dependent on $t / J$, the boundary of the holon s-wave pairing state is biased towards the lower dopings, while the boundary of the holon d-wave pairing state is moved to the higher dopings with increasing $t / J$, and we therefore find that in the underdoped and optimal doped regimes $(0.05<\delta>0.25)$ there are no in existence of the holon $\mathrm{d}$-wave pairing state for $t / J>2.3$, and the holon s-wave pairing state for $t / J>3.7$. This indicates that the holon pairing state induced by the pure magnetic interaction in the $2 \mathrm{D} t-J$ model is the local state, since the $t$ - $J$ model is characterized by a competition between the kinetic energy $(t)$ and magnetic energy $(J)$, increasing the value of 
the parameter $t / J$ means to increase the kinetic energy and tends to destroy any local state. In the overdoped case $(\delta>0.25)$, the copper oxide materials become the better metals, and are described by the Fermi-liquid theory, then the fermion-spin theory based on the chargespin separation breaks down. Therefore the above discussions about the holon pairing state are only valid for the underdoped and optimal doped regimes.

The holon pairing state originating from the magnetic interaction will also lead to form the electron pairing state. The order parameter for the electron pair is expressed as,

$$
\Delta_{\eta}=\left\langle C_{i \uparrow}^{\dagger} C_{i+\eta \downarrow}^{\dagger}-C_{i \downarrow}^{\dagger} C_{i+\eta \uparrow}^{\dagger}\right\rangle,
$$

which describes the electron Cooper pair in a range of one lattice spacing. In our present theoretical framework, the symmetry of the electron gap parameter $\Delta_{\eta}$ is determined by the symmetry of the holon gap parameter $\Delta_{\eta}^{(h)}$. For discussing the physical properties of the electron pairing state, we need to calculate the electron anomalous Green's function $\Im^{\dagger}\left(\mathbf{k}, t-t^{\prime}\right)=-\left\langle\left\langle C_{k \uparrow}^{\dagger}(t) ; C_{-k \downarrow}^{\dagger}\left(t^{\prime}\right)\right\rangle\right\rangle$, which is a convolution of the spinon Green's function $D\left(\mathbf{k}, t-t^{\prime}\right)$ and anomalous holon Green's function $\Im_{h}\left(\mathbf{k}, t-t^{\prime}\right)$ in the framework of the fermion-spin theory, i.e., $\Im^{\dagger}\left(\mathbf{k}, t-t^{\prime}\right)=(1 / N) \sum_{\mathbf{p}} D\left(\mathbf{p}, t-t^{\prime}\right) \Im_{h}\left(\mathbf{p}-\mathbf{k}, t^{\prime}-t\right)$, and can be obtained at the mean-field level as,

$$
\begin{aligned}
\Im^{\dagger}(\mathbf{k}, \omega) & =-\frac{1}{N} \sum_{p} \frac{\Delta_{h}^{(a)}(p-k)}{2 E_{p-k}} \\
& \times \frac{\Lambda\left[\left(2 \epsilon \chi_{z}+\chi\right) \gamma_{p}-\left(\epsilon \chi+2 \chi_{z}\right)\right]}{2 \omega(p)} \\
& \times\left(\frac{F_{1}(\mathbf{k}, \mathbf{p})}{\omega-\omega(p)+E_{p-k}}\right. \\
& +\frac{F_{2}(\mathbf{k}, \mathbf{p})}{\omega+\omega(p)+E_{p-k}} \\
& -\frac{F_{1}(\mathbf{k}, \mathbf{p})}{\omega+\omega(p)-E_{p-k}} \\
& \left.-\frac{F_{2}(\mathbf{k}, \mathbf{p})}{\omega-\omega(p)-E_{p-k}}\right)
\end{aligned}
$$

where $F_{1}(\mathbf{k}, \mathbf{p})=n_{B}\left(\omega_{p}\right)+n_{F}\left(E_{p-k}\right), F_{2}(\mathbf{k}, \mathbf{p})=1+$ $n_{B}\left(\omega_{p}\right)-n_{F}\left(E_{p-k}\right)$, with $n_{B}\left(\omega_{p}\right)$ and $n_{F}\left(E_{p-k}\right)$ are the spinon and holon distribution functions, respectively. In this case, the electron gap equation is obtained according to the above anomalous electron Green's function as,

$$
\begin{aligned}
\Delta^{(a)} & =-\frac{2}{N} \sum_{k} \gamma_{k}^{(a)} \frac{1}{N} \sum_{p}\left(\frac{\Delta_{h}^{(a)}(p-k)}{2 E_{p-k}} \operatorname{th}\left[\frac{1}{2} \beta E_{p-k}\right]\right) \\
& \times \frac{\Lambda\left[\left(2 \epsilon \chi_{z}+\chi\right) \gamma_{p}-\left(\epsilon \chi+2 \chi_{z}\right)\right]}{2 \omega(p)} \\
& \times \operatorname{coth}\left[\frac{1}{2} \beta \omega(p)\right]
\end{aligned}
$$

which shows that the electron gap parameter $\Delta^{(a)}$ is strong dependent on the holon gap parameter $\Delta_{h}^{(a)}$. It has been shown ${ }^{17}$ that the magnetic fluctuation is dominated by the scattering of spinons, while in the present case, this magnetic fluctuation has been incorporated into the electron anomalous Green's function (and hence the electron Cooper pair) in terms of the spinon Green's function $D(\mathbf{k}, \omega)$. Since the form of the electron Cooper pair (8) is common, and the anomalous electron Green's function always is the convolution of the spinon Green's function and anomalous holon Green's function in the framework of the fermion-spin theory, therefore there is the coexistence of the electron Cooper pair and magnetic fluctuation, and hence the AF short-range correlation can persist into the superconductivity, which is consistent with the experiments ${ }^{7}$. Moreover, we find that although there is the coexistence of the electron Cooper pair and magnetic fluctuation, the value of the electron gap parameter still suppressed by this magnetic fluctuation. Fig. 2 shows (a) the value of the electron gap parameter $\Delta^{(a)}$ at the zero temperature and (b) electron pair transition temperature $T_{c}^{(a)}$ as a function of doping $\delta$ for the s-wave symmetry (solid line) and d-wave symmetry (dashed line) in $t / J=2.0$. In comparison with Fig. 1 (a), the value of $\Delta^{(a)}$ has been decreased to about half of $\Delta_{h}^{(a)}$, while the electron pair transition temperature $T_{c}^{(s)}\left(T_{c}^{(d)}\right)$ is almost identical to the holon pair transition temperature $T_{(h) c}^{(s)}\left(T_{(h) c}^{(d)}\right)$, since according to Eq. (10), the electron pair transition temperature obtained from the condition $\Delta^{(a)}\left(T_{c}^{(a)}\right)=0$ is essential same with these from $\Delta_{h}^{(a)}\left(T_{(h) c}^{(a)}\right)=0$. It has been shown from the experiments $^{20}$ that as the doping concentration $\delta$ is reduced from the optimal doping, the superconducting gap is constant or may be slightly increasing. This reflects that the true superconducting gap is insensitive to the parameter $t / J$ in the $t-J$ model. However, in the present case the electron pairing state is dominated by the holon pairs, and as our above discussions for the properties of the holon pairing state, the present electron pairing state originating from the pure magnetic interaction in the $2 \mathrm{D}$ $t$ - $J$ model also is the local state, and then does not reveal the true superconducting ground-state, which is consistent with the numerical result obtained by Shih et al. ${ }^{21}$, they show that the pure 2D $t-J$ model does not have long-range d-wave superconducting correlation in the interesting parameter range of $t / J \geq 2$.

For the further understanding the properties of this local electron pairing state, we consider the electron spectrum function and electron density of states. According to the previous discussions ${ }^{15}$, the electron Green's function $G\left(\mathbf{k}, t-t^{\prime}\right)=\left\langle\left\langle C_{k \sigma}(t) ; C_{k \sigma}^{\dagger}\left(t^{\prime}\right)\right\rangle\right\rangle$, which is a convolution of the spinon Green's function $D\left(\mathbf{k}, t-t^{\prime}\right)$ and holon Green's function $g\left(\mathbf{k}, t-t^{\prime}\right)$, can be obtained in the present case as,

$$
G(\mathbf{k}, \omega)=\frac{1}{N} \sum_{p} \frac{\Lambda\left[\left(2 \epsilon \chi_{z}+\chi\right) \gamma_{p}-\left(\epsilon \chi+2 \chi_{z}\right)\right]}{2 \omega(p)}
$$




$$
\begin{aligned}
& \times\left\{\frac { 1 } { 2 } ( 1 + \frac { \xi _ { p - k } } { E _ { p - k } } ) \left(\frac{F_{1}(\mathbf{k}, \mathbf{p})}{\omega-\omega(p)+E_{p-k}}\right.\right. \\
& \left.+\frac{F_{2}(\mathbf{k}, \mathbf{p})}{\omega+\omega(p)+E_{p-k}}\right) \\
& +\frac{1}{2}\left(1-\frac{\xi_{p-k}}{E_{p-k}}\right)\left(\frac{F_{1}(\mathbf{k}, \mathbf{p})}{\omega+\omega(p)-E_{p-k}}\right. \\
& \left.\left.+\frac{F_{2}(\mathbf{k}, \mathbf{p})}{\omega-\omega(p)-E_{p-k}}\right)\right\} .
\end{aligned}
$$

With the help of this electron Green's function, we can obtain the electron spectrum function, $A(\mathbf{k}, \omega)=$ $-2 \operatorname{Im} G(\mathbf{k}, \omega)$ and the electron density of states $\rho(\omega)=$ $\frac{1}{N} \sum_{k} A(\mathbf{k}, \omega)$. The results of the electron spectral function and electron density of states at the doping $\delta=0.12$ for $t / J=2$ are shown in Fig. 3 (solid line) and Fig. 4 (solid line), respectively. For comparison, the results of the electron spectral function and electron density of states in the case of the electron gap parameter $\Delta=0$ at the doping $\delta=0.12$ for $t / J=2$ are also shown in Fig. 3 (dashed line) and Fig. 4 (dashed line), respectively. $\mathrm{We}^{15}$ have shown that the electron spectral function and electron density of states in the case without these local electron pairs obtained from the mean-field fermionspin theory are qualitative consistent with the numerical simulations $^{22}$ and experiments ${ }^{23}$. In the present case, although the electron spectra function and electron density of states are shifted slightly to low energy regime for

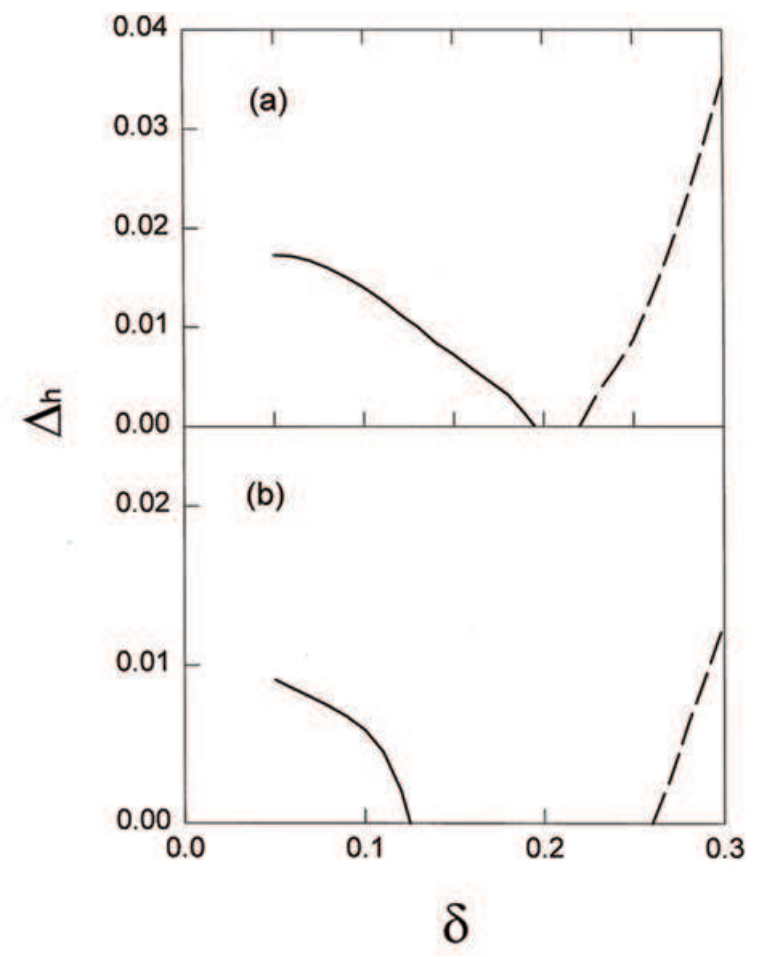

FIG. 1. The holon gap parameter in the s-wave symmetry $\Delta_{h}^{(s)}$ (solid line) and d-wave symmetry $\Delta_{h}^{(d)}$ (dashed line) as a function of doping $\delta$ at the zero temperature for (a) $t / J=2$ and (b) $t / J=2.5$. $\omega<0$, but the global feature of the electron spectral function and electron density of states is almost same with these in the case of the electron gap parameter $\Delta=0$, which shows that the normal state properties of the copper oxide materials are dominated by holons moving in the background of the spinon pair correlation. In fact, it has been shown from the experiments ${ }^{24}$ and theoretical discussions ${ }^{16,17}$ that many problems in the normal state of the copper oxide materials rely on the spinon pairing and does not require the existence of the local pairs of holons.

In summary, we have discussed the physical properties of the electron pairing state in the copper oxide materials within the fermion-spin theory. According to the common form of the electron Cooper pair, we show that there is the coexistence of the electron Cooper pair and magnetic short-range correlation, and hence the AF shortrange correlation can persist into the superconducting state, which is consistent with the experiments ${ }^{7}$. Within the mean-field level, our results indicate that the electron pairing state originating from the pure magnetic interaction in the $2 \mathrm{D} t-J$ model is the local state, and then does not reveal the true superconducting ground-state.

Finally, we note that many serious numerical studies carried out by several groups ${ }^{8,21}$ showed that at the temperatures and lattice sizes currently accessible to Monte Carlo simulations the pure 2D $t-J$ model does not super-

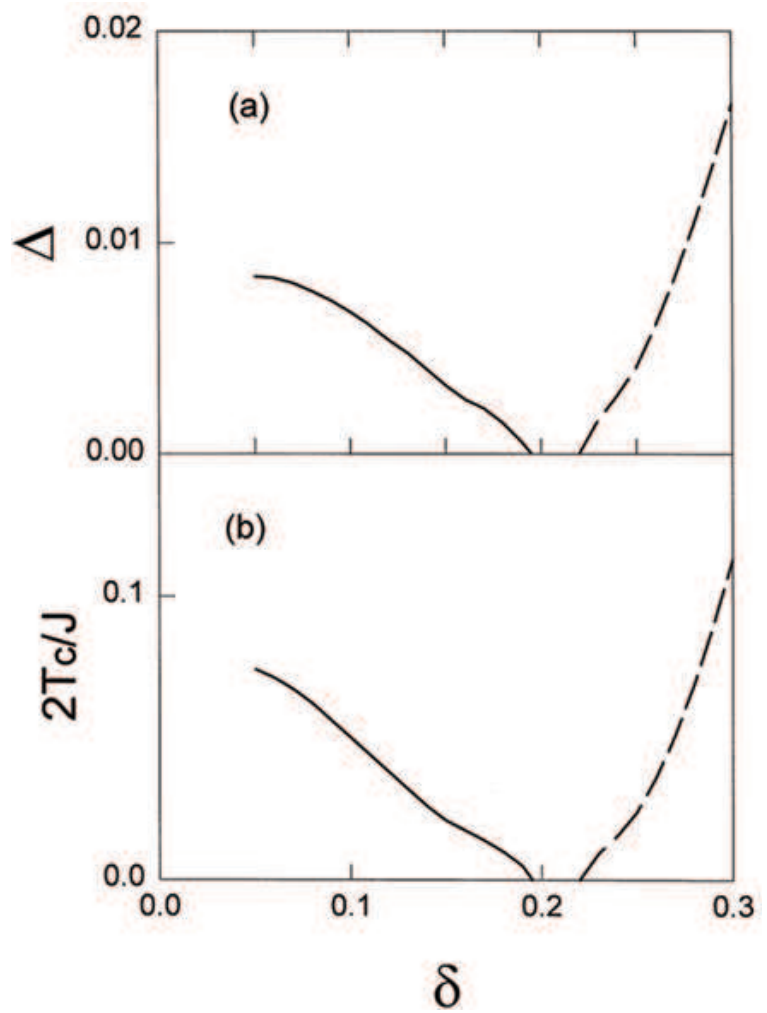

FIG. 2. (a) the electron gap parameter $\Delta$ at the zero temperature and (b) electron pair transition temperature $T_{c}$ as a function of doping $\delta$ for $t / J=2$ in the s-wave symmetry (solid line) and d-wave symmetry (dashed line). 
conduct at large $t / J$ and small hole density. Our present result is consistent with these numerical results. Since the boundary between the local electron s-wave pairing state and d-wave pairing state is changed with the parameter $t / J$, we have also found that there is a coexistence of the local electron s-wave pairing state and dwave pairing state in the underdoped and optimal doped regimes for small $t / J$, and the physical properties of this pairing state is dominated by the local electron d-wave pairing state, which is in quantitative agreement with the numerical result ${ }^{25}$. In fact, the d-wave gap function $\Delta^{(d)}(k) \propto \mathrm{k}_{x}^{2}-\mathrm{k}_{y}^{2}$ belongs to the same representation $\Gamma_{1}$ of the orthorhombic crystal group as does s-wave gap function $\Delta^{(s)}(k) \propto \mathrm{k}_{x}^{2}+\mathrm{k}_{y}^{2}$, the two perhaps can mix at will, there are some evidences from the experiments

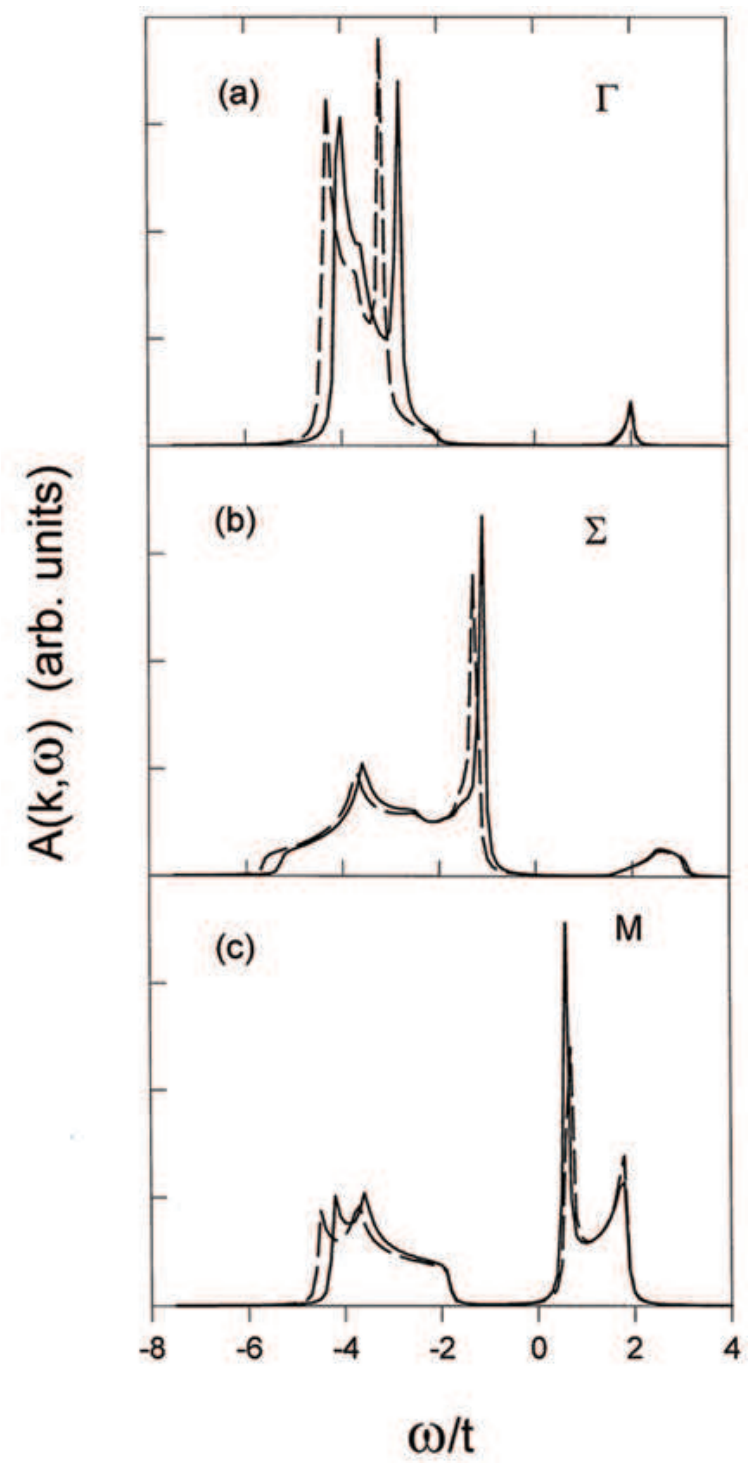

FIG. 3. The electron spectral function $A(\mathbf{k}, \omega)$ of the $t-J$ model in the cases of the existence of the local electron pairs (solid line) and without the local electron pairs (dashed line) at the doping $\delta=0.12$ for $t / J=2$. to support this symmetrical picture ${ }^{26}$. Although the normal-state is two-dimensional and coherent transport in the c-axis is blocked due to the absent of the coherent c-axis electron motion in the copper oxide materials ${ }^{27}$, it is possible that the electron Cooper pairs induced by the magnetic interaction within a given layer can tunnel between interlayers freely by the Josephson mechanism, and then the superconductivity may be motivated as the two- to three-dimensional crossover ${ }^{28}$.

\section{ACKNOWLEDGMENTS}

This work was supported by the National Natural Science Foundation under Grant No. 19774014 and the State Education Department of China through the Foundation of Doctoral Training. We thank Prof. H. Q. Lin and Prof. G. S. Tian for bringing Ref. ${ }^{21}$ to our attention.

${ }^{1}$ J. A. Martindale et al., Phys. Rev. B47, 9155 (1993); W. N. Hardy et al., Phys. Rev. Lett. 70, 3999 (1994); D. A. Wollman et al., Phys. Rev. Lett. 71, 2134 (1993).

${ }^{2}$ P. Chaudhari and S. Y. Lin, Phys. Rev. Lett. 72, 1084 (1994); D. H. Wu et al., Phys. Rev. Lett. 70, 85 (1993); S. M. Anlage et al., Phys. Rev. B44, 9764 (1991).

${ }^{3}$ P. L. Gamnel et al., Phys. Rev. Lett. 59, 2592 (1987); G. A. Thmoas et al., Phys. Rev. Lett. 61, 1313 (1988); C. E. Gough et al., Nature 326, 855 (1987); D. A. Wollman et al., Phys. Rev. Lett. 74, 797 (1995).

${ }^{4}$ Z. X. Shen et al., Phys. Rev. Lett. 70, 1553 (1993); H. Ding et al., Phys. Rev. B54, R9678 (1996).

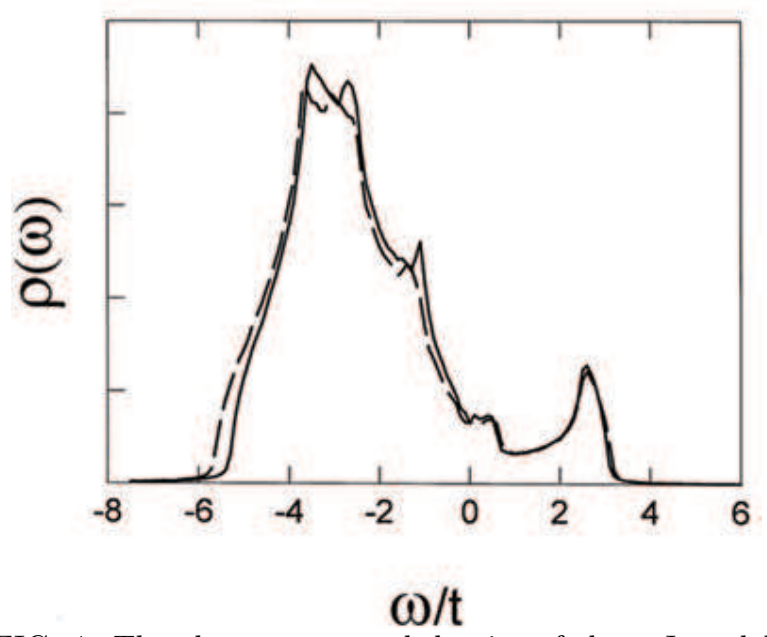

FIG. 4. The electron spectral density of the $t$ - $J$ model in the cases of the existence of the local electron pairs (solid line) and without the local electron pairs (dashed line) at the doping $\delta=0.12$ for $t / J=2$. 
${ }^{5}$ V. J. Emery et al., Phys. Rev. B56, 4111 (1997); Physica C282-287, 174 (1997).

${ }^{6}$ J. Bardeen, L. N. Cooper, and J. R. Schrieffer, Phys. Rev. 106, 162 (1957).

${ }^{7}$ A. P. Kampf, Phys. Rep. 249, 219 (1994), and references therein.

${ }^{8}$ E. Dagotto, Rev. Mod. Phys. 66, 763 (1994), and references therein.

${ }^{9}$ A. J. Millis et al., J. Phys. Chem. Solids, 56, 1641 (1995).

${ }^{10}$ P. W. Anderson, Science 235, 1196 (1987); F. C. Zhang and T. M. Rice, Phys. Rev. B 37, 3759 (1988).

11 T. M. Rice, Physica C282-287, xix (1997).

${ }^{12}$ L. Zhang et al., Phys. Rev. B47, 3368 (1993).

13 Shiping Feng et al., Phys. Rev. B 49, 2368 (1994).

${ }^{14}$ Shiping Feng et al., Mod. Phys. Lett. B10, 1301 (1996).

15 Shiping Feng and Yun Song, Phys. Rev. B55, 642 (1997).

${ }^{16}$ Shiping Feng and Zhongbing Huang, Phys. Lett. A232, 293 (1997); Zhongbing Huang and Shiping Feng, Mod. Phys. Lett B12, 735 (1998).

17 Shiping Feng and Zhongbing Huang, Phys. Rev. B57, 10328 (1998); Zhongbing Huang and Shiping Feng, Phys. Lett. A242, 94 (1998).

${ }^{18}$ S. V. Tyablikov, Methods in the Quantum Theory of Magnetism (Plenum, New York, 1967).

19 J. Kondo and K. Yamaji, Prog. Theor. Phys. 47, 807 (1972); H. Shimahara and S. Takada, J. Phys. Soc. Jpn. 60, 2394 (1991).

${ }^{20}$ A. G. Loeser et al., Science 273, 325 (1996); H. Ding et al., Nature 382, 51 (1996).

${ }^{21}$ C. T. Shih et al., Phys. Rev. Lett. 81, 1294 (1998).

22 A. Moreo et al., Phys. Rev. B 51, 12045 (1995); E. Dagotto et al., Phys. Rev. B45, 10741 (1992).

${ }^{23}$ C. T. Chen et al., Phys. Rev. Lett. 66, 104 (1991); H. Romberg et al., Phys. Rev. B 42, 8768 (1990).

${ }^{24}$ W. W. Warren et al., Phys. Rev. Lett. 62, 1193 (1989); M. Takigawa et al., Phys. Rev. B43, 247 (1991); M. Bankay et al., Phys. Rev. B50, 6416 (1994); C. C. Homes et al., Phys. Rev. Lett. 71, 1645 (1993).

${ }^{25}$ E. Dagotto and J. Riera, Phys. Rev. Lett. 70, 682 (1993).

${ }^{26}$ D. A. Brawner and H. R. Ott, Phys. Rev. B50, 6530 (1994); T. E. Mason et al., Phys. Rev. Lett. 71, 919 (1993).

${ }^{27}$ K. Tanasaki et al., Phys. Rev Lett. 69, 1455 (1992); K. Tamasaku et al., Phys. Rev. Lett. 72, 3088 (1994).

28 S. Chakravarty et al., Science 261, 337 (1993); P. W. Anderson, Science 268, 1154 (1995). 